\title{
FUZZY INSURANCE
}

\author{
By JEAN LEMAIRE \\ Wharton School \\ University of Pennsylvania, U.S.A.
}

\begin{abstract}
Fuzzy set theory is a recently developed field of mathematics, that introduces sets of objects whose boundaries are not sharply defined. Whereas in ordinary Boolean algebra an element is either contained or not contained in a given set, in fuzzy set theory the transition between membership and non-membership is gradual. The theory aims at modelizing situations described in vague or imprecise terms, or situations that are too complex or ill-defined to be analysed by conventional methods. This paper aims at presenting the basic concepts of the theory in an insurance framework. First the basic definitions of fuzzy logic are presented, and applied to provide a flexible definition of a "preferred policyholder" in life insurance. Next, fuzzy decision-making procedures are illustrated by a reinsurance application, and the theory of fuzzy numbers is extended to define fuzzy insurance premiums.
\end{abstract}

\section{KEYWORDS}

Fuzzy set theory; preferred policyholders in life insurance; optimal XLretentions; net single premiums for pure endowment insurance.

\section{INTRODUCTION}

In 1965, ZADEH published a paper entitled "Fuzzy Sets" in a little known journal, Information and Control, introducing for the first time sets of objects whose boundaries are not sharply defined. This paper gave rise to an enormous interest among researchers, and initiated the fulgurant growth of a new discipline of mathematics, fuzzy set theory. The number of papers related to the field exploded from 240 in 1975 (ZADEH et al.), to 760 in 1977 (GUPTA et al.), 2500 in 1980 (CHEN et al.), and 5000 in 1987 (ZIMMERMAN). Today, there are many more researchers in fuzzy set theory than in actuarial science, and they form a much more international group, with important contributions from China, Japan, and the Soviet Union. Two monthy scientific journals publish new theoretical developments and applications, that are to be found in linguistics, risk analysis, artificial intelligence (approximate reasoning, expert systems), pattern analysis and classification (pattern recognition, clustering, image processing, computer vision), information processing, and decisionmaking. In this paper we will explore some possible applications of fuzzy set theory to insurance.

ASTIN BULLETIN, Vol. 20, No. 1 
In ordinary Boolean algebra, an element is either contained or not contained in a given set: the transition from membership to non-membership is abrupt. Fuzzy sets, on the other hand, describe sets of elements or variables whose limits are ill-defined or imprecise. The transition between membership and non-membership is gradual: an element can "more or less" belong to a set. Consider for instance the set of "young drivers". In Boolean algebra, it is assumed that any individual either belongs or does not belong to the set of young drivers. This implies that the individual will move from the category of "young drivers" to the complementary set of " not young drivers" overnight. Fuzzy set theory allows for grades of membership. Depending on the specific application, one might for instance decide that drivers under 20 are definitely young, that drivers over 30 are definitely not young, and that a 23 -year-old driver is "more or less" young, or is young with a grade membership of 0.7 , on a scale from 0 to 1 .

Fuzzy set theory thus aims at modelizing imprecise, vague, fuzzy information, which abound in real world situations. Indeed, many practical problems are extremely complex and ill-designed, hence difficult to modelize with precision. To quote ZADEH, "as the complexity of a system increases, our ability to make precise and yet significant statements about its behaviour diminishes until a threshold is reached beyond which precision and significance become almost exclusive characteristics". Computers cannot adequately handle such problems, because machine intelligence still employs sequential (Boolean) logic. The superiority of the human brain results from its capacity of handling fuzzy statements and decisions, by adding to logic parallel and simultaneous information sources and thinking processes, and by filtering and selecting only those that are useful and relevant to its purposes. The human brain has many more thinking processes available and has developed a far greater filtering capacity than the machine. A group of individuals is able to resolve the command "tall people in the back, short people in the front", a machine is not. Fuzzy set theory explicitly introduces vagueness in the reasoning, hoping to provide decision-making procedures that are closer to the way the human brain performs.

A clear distinction has to be made between fuzzy sets and probability theory. Uncertainty should not be confused with imprecision. Probabilities are primarily intended to represent a degree of knowledge about real entities, while the degrees of membership defining the strength of participation of an entity in a class are the representation of the degree by which a proposition is partially true. Probability concepts are derived from considerations about the uncertainty of propositions about the real world. Fuzzy concepts are closely related to the multivalued logic treatments of issues of imprecision in the definition of entities. Hence, fuzzy set theory provides a better framework than probability theory for modelling problems that have some inherent imprecision. The traditional approach to risk analysis, for instance, is based on the premise that probability theory provides the necessary and sufficient tools for dealing with the uncertainty and imprecision which underline the concept of risk in decision analysis. The theory of fuzzy sets calls into question the validity of this 
premise. It does not equate imprecision with randomness. It suggests that much of the uncertainty which is intrinsic in risk analysis is rooted in the fuzziness of the information which is resident in the data base and in the imprecision of the underlying probabilities. Classical probability theory has its effectiveness limited when dealing with problems in which some of the principal sources of uncertainty are non-statistical in nature.

In the sequel we will present the basic principles of fuzzy logic, fuzzy decision-making, and fuzzy arithmetics, while developing three insurance examples. We will show that fuzzy set theory could provide decision procedures that are much more flexible than those originating from conventional set theory. Indeed, insurance executives and actuaries, much better trained to deal with uncertainty than with vagueness, have often transformed imprecise statements into "all-or-nothing" rules. For instance, Belgian insurers have used the fuzzy statistical evidence "Young drivers provoke more automobile accidents" to set up the a posteriori rating rule "Drivers under 23 years of age will pay a $\$ 150$ deductible if they provoke an accident". Hence "young" was equated with "under 23 ", a definite distorsion of the initial statement. As another example, Belgian regulatory authorities define, for statistical purposes, a "severely wounded person" as "any person, wounded in an automobile accident, whose condition requires a hospital stay longer than 24 hours", a very arguable "de-fuzzification" of a fuzzy health condition.

In Section 2 we will present the basic definitions of fuzzy logic and apply them to provide a more flexible definition of a "preferred policyholder" than the one currently used by some American life insurers. Section 3 introduces the main concepts of fuzzy decision-making, and uses them to select an optimal Excess of Loss retention. Fuzzy arithmetics are presented in Section 4, and applied to compute the fuzzy premium of a pure endowment policy.

First, let us introduce our three examples.

\section{Problem 1: Definition of a preferred policyholder in life insurance}

Heavy competition between American life insurers has resulted in a greater subdivison of policyholders than in Europe. U.S. insurers first began, in the mid 1960 s, to award substantial discounts to nonsmokers purchasing a term or a whole life insurance. Then the "preferred policyholder" category was further refined, and more discounts were granted to applicants who met very stringent health requirements, such as a cholesterol level not exceeding 200, a blood pressure not exceeding 130/80,... For instance, one company offers a nonsmoker bonus of $65 \%$ more insurance coverage with no increase in premium if the applicant has not smoked for 12 months prior to application. A bonus of $100 \%$ is offered if the applicant :

- has not smoked for the past 12 months, and

- has a resting pulse of 72 or below, and

- has a blood pressure that does not exceed 134/80, and

- has a total cholesterol reading not exceeding 200 , and 
- does not engage in hazardous sports, and

- rigorously follows a 3-times-a-week exercise program of at least 20 minutes, and

- is within specified height and weight limits, and

- has no more than one death in immediate family prior to 60 years of age due to kidney or heart disease, stroke or diabetes.

Again this is a distorsion, or a least a very strict interpretation, of the medical statement "People who exercise, who do not smoke, who have a low level of cholesterol, low blood pressure, who are neither overweight nor severely underweight, ... have a higher life expectancy ". Insurers demand all conditions to be strictly met; the slightest infringement leads to automatic rejection of the preferred category. For instance, a cholesterol level of 201 implies that the preferred rates won't apply, even if the applicant meets all other requirements. A cholesterol level of 200 is accepted, a level of 201 is not! We will show that fuzzy set theory can be used to provide a more flexible definition of a preferred policyholder, that allows for some form of compensation between the selected criteria.

\section{Problem 2: Selection of an optimal excess of loss retention}

Imprecise statements seem to be pervasive in reinsurance practive, where vague recommendations and rules abound. "As a rule of thumb, an excess of loss (XL) retention should approximatively equal $1 \%$ of the premium income", "Our long-term relationship with our present reinsurer should in principle be maintained", "We could accept those conditions providing substantial retrocessions are offered", "A ball-park figure for the cost of this reinsurance program is $\$ 10$ million ", are fuzzy sentences frequently heard in practice. To illustrate fuzzy decision-making procedures, we shall consider the problem of the selection of the optimal retention of a pure XL treaty, given the four following fuzzy goals and constraints.

Goal 1: The ruin probability should be substantially decreased, ideally down to be neighbourhood of $10^{-5}$.

Goal 2: The coefficient of variation of the retained portfolio should be reduced; if possible it should not exceed 3.

Constraint 1: The reinsurance premium should not exceed $2.5 \%$ of the line's premium income by much.

Constraint 2: As a rule of thumb, the retention should approximatively be equal to $1 \%$ of the line's premium income.

\section{Problem 3: Computation of the fuzzy premium of a pure endowment policy}

Forecasting interest rates is undoubtedly one of the most complex modelling problems. Money market interest rates seem to fluctuate according to monthly U.S. unemployment and trade deficit figures, vague statements made by Mr Kohl or Mr Greenspan, the markets' perception of Mr Bush's willingness to tackle the deficit problem, the mood of the participants to an OPEC 
meeting, etc. To compute insurance premiums over a 40 -year span with a fixed interest rate of $4.75 \%$ then seems to be an exercise in futility. We will show that the introduction of fuzzy interest rates (and fuzzy survival probabilities) at least allows us to obtain a partial measure of our ignorance.

As illustrated by our examples, fuzzy set theory attempts to modelize imprecise expressions like "more or less young", "neither overweight nor underweight", "in the neighbourhood of", "in principle". In retreating from precision in the face of overpowering complexity, the theory explores the use of what might be called linguistic variables, that is, variables whose values are not numbers but words or sentences. In summary, fuzzy set theory endorses Bertrand Russell's opinion that

"All traditional logic habitually assumes that precise symbols are being employed. It is therefore not applicable to this terrestrial life but only to an imagined celestial existence"

and rejects Yves Le Dantec's aphorism

"That only is science which deals with the measurable".

\section{FUZZY LOGIC AND FUZZY PREFERRED POLICYHOLDERS}

\subsection{Basic definitions}

A fuzzy set is a class of objects in which there is no sharp boundary between those objects that belong to the class and those that do not. More precisely, let $X=\{x\}$ denote a collection of objects denoted generically by $x$. A fuzzy set $A$ in $X$ is a set of ordered pairs

$$
A=\left\{x, U_{A}(x)\right\}, \quad x \in X
$$

where $U_{A}(x)$ is termed the grade of membership of $x$ in $A$, and $U_{A}: X \rightarrow M$ is a function from $X$ to a space $M$, called the membership space. Hence a fuzzy set $A$ on a referential set $X$ can be viewed as a mapping $U_{A}$ from $X$ to $M$. (Examples of membership functions are presented in all figures).

For our purposes it is sufficient to assume that $M$ is the interval $[0,1]$, with 0 and 1 representing, respectively, the lowest and highest grade of membership. The degree of membership of $x$ in $A$ corresponds to a "truth value" of the statement " $x$ is a member of $A$ ". When $M$ only contains the two points 0 and $1, A$ is nonfuzzy.

\section{Problem 1}

Let $X$ be a set of prospective policyholders, $x=x\left(t_{1}, t_{2}, t_{3}, t_{4}\right)$. For simplicity, assume that the requirements for the status of "preferred policyholder" will be based on the values taken by 4 variables

$t_{1}$, the total level of cholesterol in the blood, in $\mathrm{mg} / \mathrm{dl}$,

$t_{2}$, the systolic blood pressure, in $\mathrm{mm}$ of $\mathrm{Hg}$ 
$t_{3}$, the ratio (in \%) of the effective weight to the recommended weight, as a function of height and build

$t_{4}$, the average consumption of cigarettes per day.

Using a classical approach, an insurance company would for instance define a preferred policyholder as a nonsmoker with a cholesterol level that does not exceed 200 , and a blood pressure that does not exceed 130 , and a weight that is comprised between $85 \%$ and $110 \%$ of his recommended weight.

If a fuzzy set approach is to be used, membership functions have to be defined for all criteria.

National Institutes of Health nowadays recommend a level of less than $200 \mathrm{mg}$ of cholesterol per deciliter of blood. Levels between 200 and $240 \mathrm{mg} / \mathrm{dl}$ are considered to be borderline high. The fuzzy set $A$ of the people with a low level of cholesterol can then be defined by the membership function $U_{A}\left(x ; t_{1}\right)$

$$
U_{A}\left(x ; t_{1}\right)=\left\{\begin{array}{lr}
1 & t_{1} \leqq 200 \\
1-2\left(\frac{t_{1}-200}{40}\right)^{2} & 200<t_{1} \leqq 220 \\
2\left(\frac{240-t_{1}}{40}\right)^{2} & 220<t_{1} \leqq 240 \\
0 & 240<t_{1}
\end{array}\right.
$$

The normal systolic blood pressure is about $130 \mathrm{~mm}$ of mercury. People with a blood pressure greater than 170 are five times more likely to suffer from coronary heart disease than individuals with normal blood pressures. Hence the fuzzy set $B$ of the people with an acceptable blood pressure can be defined by the membership function $U_{B}\left(x ; t_{2}\right)$

$$
U_{B}\left(x ; t_{1}\right)=\left\{\begin{array}{lr}
1 & t_{2} \leqq 130 \\
1-2\left(\frac{t_{2}-130}{40}\right)^{2} & 130<t_{2} \leqq 150 \\
2\left(\frac{170-t_{2}}{40}\right)^{2} & 150<t_{2} \leqq 170 \\
0 & 170<t_{2}
\end{array}\right.
$$

Overweight and underweight people have a shorter life expectancy, skinniness being less primordial than obesity. This is reflected in the asymmetric membership function $U_{C}\left(x ; t_{3}\right)$ that characterizes the fuzzy set $C$ of the people with adequate weight. 


$$
U_{C}\left(x ; t_{3}\right)=\left\{\begin{array}{lr}
0 & t_{3} \leqq 60 \\
2\left(\frac{t_{3}-60}{25}\right)^{2} & 60<t_{3} \leqq 72.5 \\
1-2\left(\frac{85-t_{3}}{25}\right)^{2} & 72.5<t_{3} \leqq 85 \\
1-2\left(\frac{t_{3}-110}{20}\right)^{2} & 85<t_{3} \leqq 110 \\
2\left(\frac{130-t_{3}}{20}\right)^{2} & 110<t_{3} \leqq 120 \\
0< & 120<t_{3} \leqq 130
\end{array}\right.
$$

Even light smokers are more prone to suffer from cancer and cardiovascular diseases than nonsmokers. Hence they cannot be considered as "preferred" and the set $D$ of the nonsmokers is nonfuzzy

$$
U_{D}\left(x ; t_{4}\right)= \begin{cases}1 & t_{4}=0 \\ 0 & t_{4}>0 .\end{cases}
$$

The four selected membership functions are represented in Figure 1. Admittedly, there is some arbitrariness in the definiton of these membership functions, but fuzzy set theory contends that this is better than membership functions that abruptly jump from 1 to 0 , in the classical approach.

A fuzzy set is said to be normal iff $\operatorname{Sup}_{x} U_{A}(x)=1$. Subnormal fuzzy sets can be normalized by dividing each $U_{A}(x)$ by the factor $\operatorname{Sup}_{x} U_{A}(x)$.

$\bar{A}$ is said to be the complement of $A$ iff $U_{\bar{A}}(x)=1-U_{A}(x) \quad \forall x$.

A fuzzy set is contained in or is a subset of a fuzzy set $B(A \subset B)$ iff $U_{A}(x) \leqq U_{B}(x) \quad \forall x$.

The union of $A$ and $B$, denoted $A \cup B$, is defined as the smallest fuzzy set containing both $A$ and $B$. Its membership function is given by

$$
U_{A \cup B}(x)=\max \left[U_{A}(x), U_{B}(x)\right] \quad x \in X
$$

The intersection of $A$ and $B$, denoted $A \cap B$, is defined as the largest fuzzy set contained in both $A$ and $B$. Its membership function is given by

$$
U_{A \cap B}(x)=\min \left[U_{A}(x), U_{B}(x)\right] \quad x \in X
$$

The notion of intersection bears a close relation to the notion of the connective "and", just as the union of $A$ and $B$ bears a close relation to the connective "or". It can be shown that these definitions of fuzzy union and intersection are 

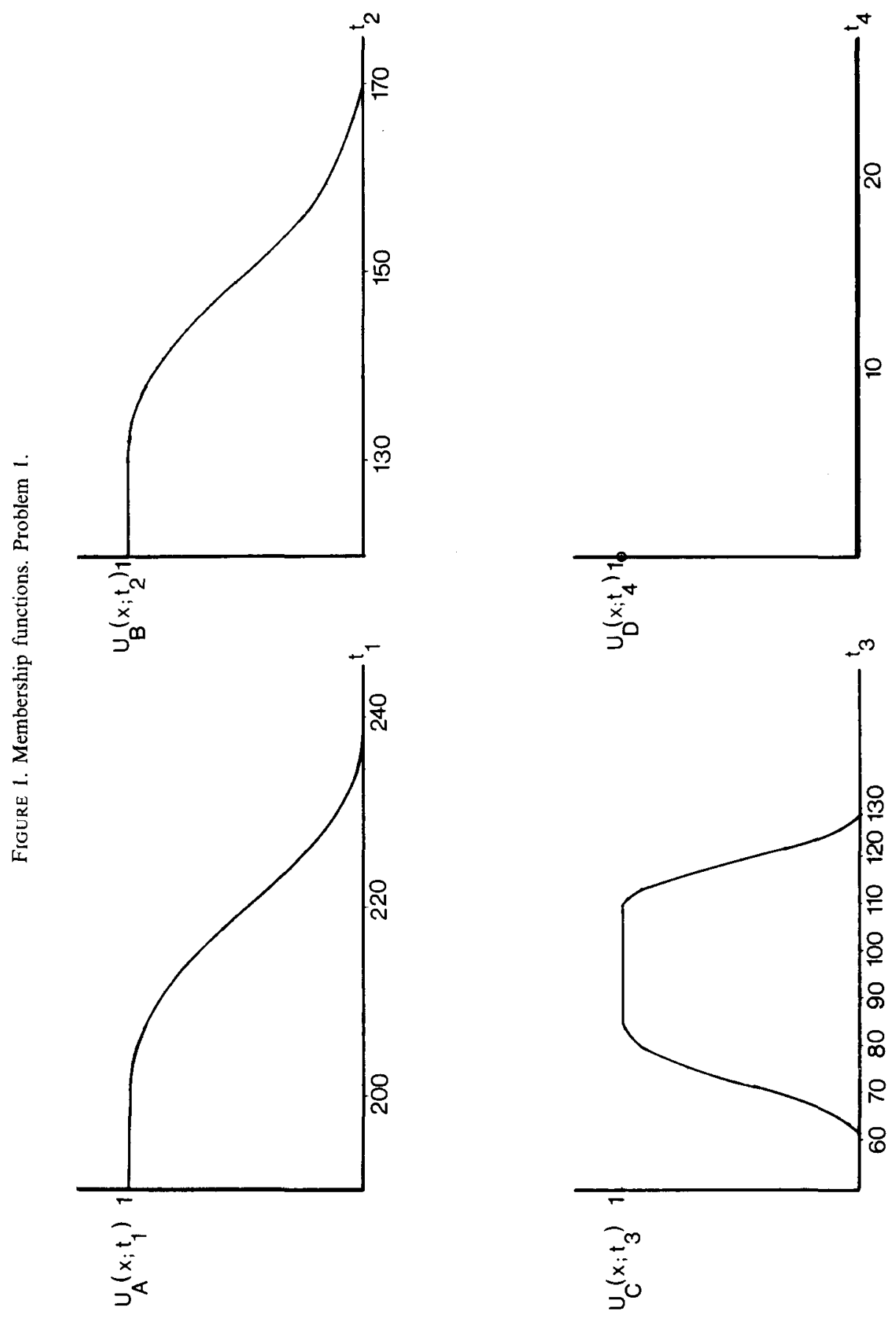
the only ones that naturally extend the corresponding standard set theory notions, by satisfying all the usual requirements of associativity, commutativity, idempotency and distributivity.

\section{Problem 1}

The fuzzy set $E$ of the nonsmoking individuals with low cholesterol, acceptable blood pressure and adequate weight is the intersection of the 3 fuzzy sets $A, B$, $C$, and the nonfuzzy set $D$. Its membership function is given by

$$
U_{E}\left(x ; t_{1}, t_{2}, t_{3}, t_{4}\right)=\min \left[U_{A}\left(x ; t_{1}\right), U_{B}\left(x ; t_{2}\right), U_{C}\left(x ; t_{3}\right), U_{D}\left(x ; t_{4}\right)\right]
$$

So an individual can only be a full member of $E$ if he doesn't smoke, has a cholesterol level not exceeding 200, a blood pressure not above 130, and a weight no less than $85 \%$ and no more than $110 \%$ of his recommended or ideal weight. This corresponds to the classical approach.

A nonsmoker $x=x(210,145,112,0)$ with a cholesterol level of 210 , a blood pressure of 145 , and who is overweight by $12 \%$ is a member of $E$ with a grade of membership

$$
U_{E}(x ; 210,145,112,0)=\min (0.875,0.71875,0.98,1)=0.71875 .
$$

In other words, the " $\cap$ " operation assigns a grade of membership that corresponds to the most severe of the infringements to "perfection", in this case blood pressure. Cumulative effects and interactions between the criteria are ignored, which is not realistic. Obviously, the health consequences of high blood pressure are worse when there is also an excess of weight and cholesterol. Also, since only the most severe condition is considered, it is impossible to introduce compensations or trade-offs in decision rules. A mild excess of weight cannot be compensated by ideal cholesterol and blood pressure.

\subsection{Other definitions of the intersection}

The minimum operator that characterizes the intersection corresponds to the "logical and". Other definitions of the intersection have been suggested; they correspond to "softer", more flexible interpretations of the connective "and". They all amount to exactly the same in the conventional case of degrees of membership restricted to 0 and 1 . The selection of a specific operator will depend on its possibilities to allow for cumulative effects, interactions, and compensations between the criteria. We wish the following properties to be satisfied.

Property 1 (cumulative effects): Two infringements are worse than one.

$$
U_{A \cap B}(x)<\min \left[U_{A}(x), U_{B}(x)\right] \text { if } U_{A}(x)<1 \text { and } U_{B}(x)<1 .
$$

Property 2 (interactions between criteria): Assume $U_{A}(x)<U_{B}(x)<1$. Then the effect of a decrease of $U_{A}(x)$ on $U_{A \cap B}(x)$ may depend on $U_{B}(x)$. 
Property 3 (compensations between criteria): If $U_{A}(x)$ and $U_{B}(x)<1$, the effect of a decrease of $U_{A}(x)$ on $U_{A \cap b}(x)$ can be erased by an increase of $U_{B}(x)$ (unless, of course, $U_{B}(x)$ reaches 1 ).

The algebraic product $F$ of $A$ and $B$ is denoted $A B$ and is defined by

$$
U_{A B}(x)=U_{A}(x) \cdot U_{B}(x)
$$

The bounded difference $G$ of $A$ and $B$ is denoted $A \ominus B$ and is defined by

$$
U_{A \ominus B}(x)=\max \left[0, U_{A}(x)+U_{B}(x)-1\right]
$$

The Hamacher operator $H$ defines the intersection of two fuzzy sets $A$ and $B$ by

$$
U_{H}^{p}(x)=\frac{U_{A}(x) \cdot U_{B}(x)}{p+(1-p)\left[U_{A}(x)+U_{B}(x)-U_{A}(x) U_{B}(x)\right]} \quad 0 \leqq p \leqq 1
$$

The Yager operator $Y$ defines the intersection of two fuzzy sets $A$ and $B$ by

$$
U_{Y}^{p}(x)=1-\min \left\{1,\left[\left(1-U_{A}(x)\right)^{p}+\left(1-U_{B}(x)\right)^{p}\right]^{1 / p}\right\} \quad p \geqq 1
$$

\section{Problem 1}

The generalized operators provide a more realistic way of modelling this specific problem because they explicitly allow for compensations and interactions between the selected criteria. First consider the algebraic product. The grade of membership of individual $x(210,145,112,0)$ in the fuzzy set $F=A B C D$ is

$$
U_{F}(x ; 210,145,112,0)=(0.875)(0.71875)(0.98)(1)=0.6163 .
$$

The effect of high blood pressure is here amplified by the presence of a slight obesity and a cholesterol level mildly above normal. This operator satisfies all three properties.

The grade of membership of the same individual in the fuzzy set $G=A \ominus B \ominus C \ominus D$ corresponding to the bounded difference operation is

$$
U_{G}(x ; 210,145,112,0)=\max [0,0.875+0.71875+0.98+1-3]=0.57375 .
$$

Hence the effects of the criteria are additive; no interactions are introduced, since the consequences of cholesterol are the same whatever the blood pressure and the weight. This operator satisfies properties 1 and 3 , but not property 2.

The minimum and algebraic product operators model two extreme situations. The minimum operator does not satisfy any property. Compensations and interactions cannot be introduced. The algebraic product allows for compensation and maximum interaction, since the effect of one criterion fully impacts the others. The Hamacher and Yager operators model intermediate situations, with flexibility provided by the parameter $p$.

The Hamacher operator reduces to the algebraic product when $p=1$. For $p<1$, the denominator is less than 1 and $U_{H}(x)>U_{F}(x)$ : the product 
operator is "softened"; this operator models weaker interactions. It reduces the effect of combined infringements. The reduction effect is greater for severe infringements. Also, the lower the selected $p$, the greater the reduction effect. Hence this operator can be used if it is considered that the combined effect of high cholesterol and high blood pressure is somewhat less than multiplicative. Selecting $p=0.5$ for our example, we obtain successively

$$
\begin{gathered}
U_{H}^{1 / 2}(x ; 210,145)=\frac{(0.875)(0.71875)}{0.5+(1-0.5)[0.875+0.71875-(0.875)(0.71875)]}=0.6402 \\
U_{H}^{1 / 2}(x ; 210,145,112,0)=U_{H}^{1 / 2}(x ; 210,145,112) \\
=\frac{(0.6402)(0.98)}{0.5+(1-0.5)[0.6402+0.98-(0.6402)(0.98)]}=0.6296
\end{gathered}
$$

This operator satisfies all three properties.

The Yager operator reduces to the bounded difference operator when $p=1$, and to the minimum operator when $p \rightarrow \infty$. $U_{Y}^{p}(x)$ is an increasing function of $p$. Hence all intermediate situations can be modelled, from the strongest to the weakest "and". Selecting $p=2$, we obtain

$$
U_{Y}^{2}(x)=1-\min \left\{1,\left[(1-0.875)^{2}+(1-0.71875)^{2}+(1-0.98)^{2}\right]^{1 / 2}\right\}=0.69157 .
$$

This operator satisfies all three properties, except in the case $p=\infty$.

\subsection{Selection of a decision rule}

If $A$ is a fuzzy subset of $X$, its $a$-cut $A_{a}$ is defined as the nonfuzzy subset such that

$$
A_{a}=\left\{x \mid U_{a}(x) \geqq a\right\} \quad \text { for } \quad 0<a \leqq 1 .
$$

An $a$-cut can be interpreted as an error interval whose truth value is $a$.

\section{Problem 1}

The notion of $a$-cut provides a flexible way of defining preferred policyholders. The "classical" approach corresponds to 1-cuts such as $E_{1}$ or $F_{1}$. Lower values of $a$ provide generalizations of this definition. For instance preferred customers could be defined as the members of $E_{0.75}$ or $F_{0.60} . E_{0.75}$ is the set of policyholders for which the grade of membership attains at least 0.75 for each of the selected criteria (for our specific membership functions, $t_{1} \leqq 214$, $t_{2} \leqq 144,76.2<t_{3} \leqq 117.1, t_{4}=0$ ). Hence this amounts to relaxing all criteria in a uniform way.

$F_{0.60}$ is the set of policyholders for which the product of the four grades of membership attains at least 0.60 . The latter definition is more realistic because it allows for interactions and compensations. An excess of blood pressure can for instance be compensated by normal or near-normal weight and cholesterol 
levels. Policyholder $x(210,145,112,0)$ is accepted as preferred using the second criterion. He is not accepted if the first criterion is used.

Similar decision rules can be constructed using the other operators, if medical considerations hint that they provide a better model of the problem.

\subsection{Fuzzy operations}

The concept of grades of membership allows to define the following operations that have no counterpart in ordinary set theory; they are uniquely fuzzy.

Concentration: A fuzzy set is concentrated by reducing the grade of membership of all elements that are only partly in the set, in such a way that the less an element is in the set, the more its grade of membership is reduced. The concentration of a fuzzy set $A$ is denoted $\operatorname{CON}(A)$ and defined by

$$
U_{\mathrm{CON}(A)}(x)=U_{A}^{a}(x) \quad a>1
$$

Dilation: Dilation is the opposite of concentration. A fuzzy set is dilated or stretched by increasing the grade of membership of all elements that are partly in the set. The dilation of a fuzzy set $A$ is denoted DIL $(A)$ and defined by

$$
U_{\mathrm{DIL}(A)}(x)=U_{A}^{a}(x) \quad a<1
$$

$a$ is called the power of the operation.

Intensification: A fuzzy set can be intensified by increasing the grade of membership of all the elements that are at least half in the set and decreasing the grade of membership of the elements that are less than half in the set. The intensification of a fuzzy set is denoted INT $(A)$ and is defined by

$$
U_{\mathrm{INT}(A)}(x)= \begin{cases}2 U_{A}^{2}(x) & 0<U(x) \leqq 0.5 \\ 1-2\left[1-U_{A}(x)\right]^{2} & 0.5<U(x) \leqq 1\end{cases}
$$

Fuzzification: A fuzzy set can be fuzzified or de-intensifed by increasing the extent of its fuzziness. There are several ways of achieving this.

\section{Problem 1}

The operations of concentration and dilation roughly approximate the effect of the linguistic modifiers "very" and "more or less". They are used whenever the different criteria have to be weighted. The presentation of problem 1 so far implicitly assumes that each criterion has the same importance. If for medical reasons this is not desirable, fuzzy operations can be used. Suppose that cholesterol level is the better predictor of future heart problems, while the importance of blood pressure has to be downgraded. This can be reflected by 
assigning powers of 2 and 0.5 to the two criteria. The modified fuzzy set $\widetilde{E}$, corresponding to the minimum operator, is characterized by

$$
U_{\tilde{E}}\left(x ; t_{1}, t_{2}, t_{3}, t_{4}\right)=\min \left[U_{A}^{2}\left(x ; t_{1}\right), U_{B}^{1 / 2}\left(x ; t_{2}\right), U_{C}\left(x ; t_{3}\right), U_{D}\left(x ; t_{4}\right)\right]
$$

The modified fuzzy set $\tilde{F}$, corresponding to the algebraic product, has the membership function

$$
U_{\tilde{F}}\left(x ; t_{1}, t_{2}, t_{3}, t_{4}\right)=U_{A}^{2}\left(x ; t_{1}\right) U_{B}^{1 / 2}\left(x ; t_{2}\right) U_{C}\left(x ; t_{3}\right) U_{D}\left(x ; t_{4}\right)
$$

Prospective policyholder $x(210,145,112,0)$ has a grade of membership of

$$
\min \left[(0.875)^{2},(0.71875)^{1 / 2}, 0.98,1\right]=0.7656
$$

in $\tilde{E}$, and of

$$
(0.875)^{2} \cdot(0.71875)^{1 / 2} \cdot(0.98) \cdot(1)=0.6361
$$

in $\tilde{F}$. He is now accepted as a preferred customer under each of the two criteria of Section 2.3 , since $x(210,145,112,0)$ is included in both $\widetilde{E}_{0.75}$ and $\widetilde{F}_{0.60}$.

\section{DECISION-MAKING WITH FUZZY GOALS AND CONSTRAINTS AND FUZZY REINSURANCE}

In the classical approach to decision-making, the principal ingredients of a decision problem are $(a)$ a set of alternatives, $(b)$ a set of constraints on the choice between different alternatives, and $(c)$ an objective function which associates with each alternative its evaluation. There is however an intrinsic similarity between objective functions and constraints, a similarity that becomes apparent when for instance Lagrangian multipliers are introduced.

This similarity is made explicit in the formulation of a decision problem in a fuzzy environment. Let $X=\{x\}$ be a given set of alternatives. A fuzzy goal $G$ in $X$, or simply a goal $G$, is expressed and identified with a given fuzzy set $G$ in $X$. In other words, a fuzzy goal is an objective which can be characterized as a fuzzy set in the space of alternatives. In the classical approach, the objective function serves to define a linear ordering on the set of alternatives. Clearly the membership function $U_{G}(x)$ of a fuzzy goal serves the same purpose, and may even be derived from a given objective function by normalization, which leaves the linear ordering unaltered. Such normalization provides a common denominator for the various goals and constraints and makes it possible to treat them alike. A fuzzy constraint $C$ in $X$, or simply a constraint $C$, is similarly defined to be a fuzzy set $C$ in $X$. An important aspect of those definitions is thus that the notions of goal and constraint both are defined as fuzzy sets in the space of alternatives. Hence they can be treated identically in the decision process. Since we want to satisfy (optimize) the objective function as well as the constraints, a decision in a fuzzy environment is defined as the selection of activities which simultaneously satisfy objective functions and constraints. A decision can therefore be viewed as the intersection of fuzzy constraints and fuzzy objective function(s). The relationship between constraints and objective functions in a fuzzy environment is therefore fully symmetric. 
Assume we are given a finite set of alternatives $X=\left\{x_{1}, x_{2}, \ldots, x_{n}\right\}$, a set of goals $G_{1}, \ldots, G_{p}$, characterized by their respective membership functions $U_{G_{1}}(x), \ldots, U_{G_{p}}(x)$, and a set of constraints $C_{1}, \ldots, C_{q}$, characterized by their respective membership functions $U_{C_{1}}(x), \ldots, U_{C_{g}}(x)$. Finiteness is assumed for expository purposes only and can be easily relaxed.

A decision is a choice or a set of choices drawn from the available alternatives, satisfying the constraints and the goals. The constraints and goals combine to form a decision $D$, which is naturally defined as the intersection of the fuzzy sets $G$ 's and $C$ 's.

$$
D=G_{1} \cap G_{2} \cap \ldots \cap G_{p} \cap C_{1} \cap C_{2} \cap \ldots \cap C_{q}
$$

Consequently a decision $D$ is a fuzzy set in the space of alternatives whose membership function is

$$
U_{D}(x)=\min \left[U_{G_{1}}(x), \ldots, U_{G_{p}}(x), U_{C_{1}}(x), \ldots, U_{C_{q}}(x)\right]
$$

This decision membership function can be interpreted as the degree to which each of the alternatives satisfies the goals and constraints. As in example 1, concentrations and dilations can be performed to reflect unequal importances of the goals and constraints, and other intersection operators can be used.

Let $K$ be the (nonfuzzy) set consisting of all the alternatives for which $U_{D}(x)$ reaches its maximal value. $K$ is called the optimizing set, and any alternative in $K$ is an optimal decision. The decision-maker simply selects as best alternative the one that has the maximum value of membership in $D$.

This decision-making procedure is essentially a maximin technique, similar to the selection of an optimal strategy in noncooperative game theory. For each alternative the minimum possible grade of membership of all the goals and constraints is computed to obtain $D$. Then the maximum value over the alternatives in $D$ is selected.

\section{Problem 2}

Given the formulation of the problem, a reinsurance program is characterized by its XL deductible, and evaluated by means of 4 different variables

$t_{1}=$ probability of ruin $\left(\times 10^{4}\right)$

$t_{2}=$ coefficient of variation of the retained portfolio

$t_{3}=\frac{\text { reinsurance premium }}{\text { cedent's premium income }}$ (in \%)

$t_{4}=\frac{\text { deductible }}{\text { cedent's premium income }}$ (in \%)

Assume the reinsurer offers 10 different XL deductibles, arranged in increasing order $(x=1,2, \ldots, 10)$. The values taken by the selected variables are provided in Table 1. 
TABLE 1

Characteristics of the 10 XL reinsurance programs

\begin{tabular}{|c|c|c|c|c|c|c|c|c|c|c|}
\hline Program & 1 & 2 & 3 & 4 & 5 & 6 & 7 & 8 & 9 & 10 \\
\hline$G_{1} \quad t_{1}$ & .339 & .280 & .200 & .200 & .313 & .339 & .360 & .388 & .419 & .465 \\
\hline$G_{2} \quad t_{2}$ & 2.98 & 3.00 & 3.03 & 3.07 & 3.12 & 3.19 & 3.28 & 3.52 & 3.80 & 4.20 \\
\hline$C_{1} t_{3}$ & 3.20 & 3.00 & 2.85 & 2.73 & 2.64 & 2.57 & 2.52 & 2.48 & 2.45 & 2.43 \\
\hline$C_{2} t_{4}$ & .4 & .6 & .8 & .9 & 1.0 & 1.1 & 1.2 & 1.4 & 1.6 & 1.8 \\
\hline
\end{tabular}

The following membership functions have been chosen. They are represented in Figure 2.

Goal 1 (probability of ruin)

$$
U_{G_{l}}\left(x ; t_{1}\right)=\left\{\begin{array}{lr}
1 & t_{1} \leqq .00002 \\
1-2\left(\frac{t_{1}-.00002}{.00008}\right)^{2} & .00002<t_{1} \leqq .00006 \\
2\left(\frac{.0001-t_{1}}{.00008}\right)^{2} & .00006<t_{1} \leqq .0001 \\
0 & .0001<t_{1}
\end{array}\right.
$$

Goal 2 (coefficient of variation)

$$
U_{G_{2}}\left(x ; t_{2}\right)=\left\{\begin{array}{lr}
1 & t_{2} \leqq 3.1 \\
4.1-t_{2} & 3.1<t_{2} \leqq 4.1 \\
0 & 4.1<t_{2}
\end{array}\right.
$$

Constraint 1 (reinsurance premium)

$$
U_{C_{1}}\left(x ; t_{3}\right)=\left\{\begin{array}{lr}
1 & t_{3} \leqq 2.5 \\
1-2\left(\frac{t_{3}-2.5}{0.6}\right)^{2} & 2.5<t_{3} \leqq 2.8 \\
2\left(\frac{3.1-t_{3}}{0.6}\right)^{2} & 2.8<t_{3} \leqq 3.1 \\
0 & 3.1<t_{3}
\end{array}\right.
$$



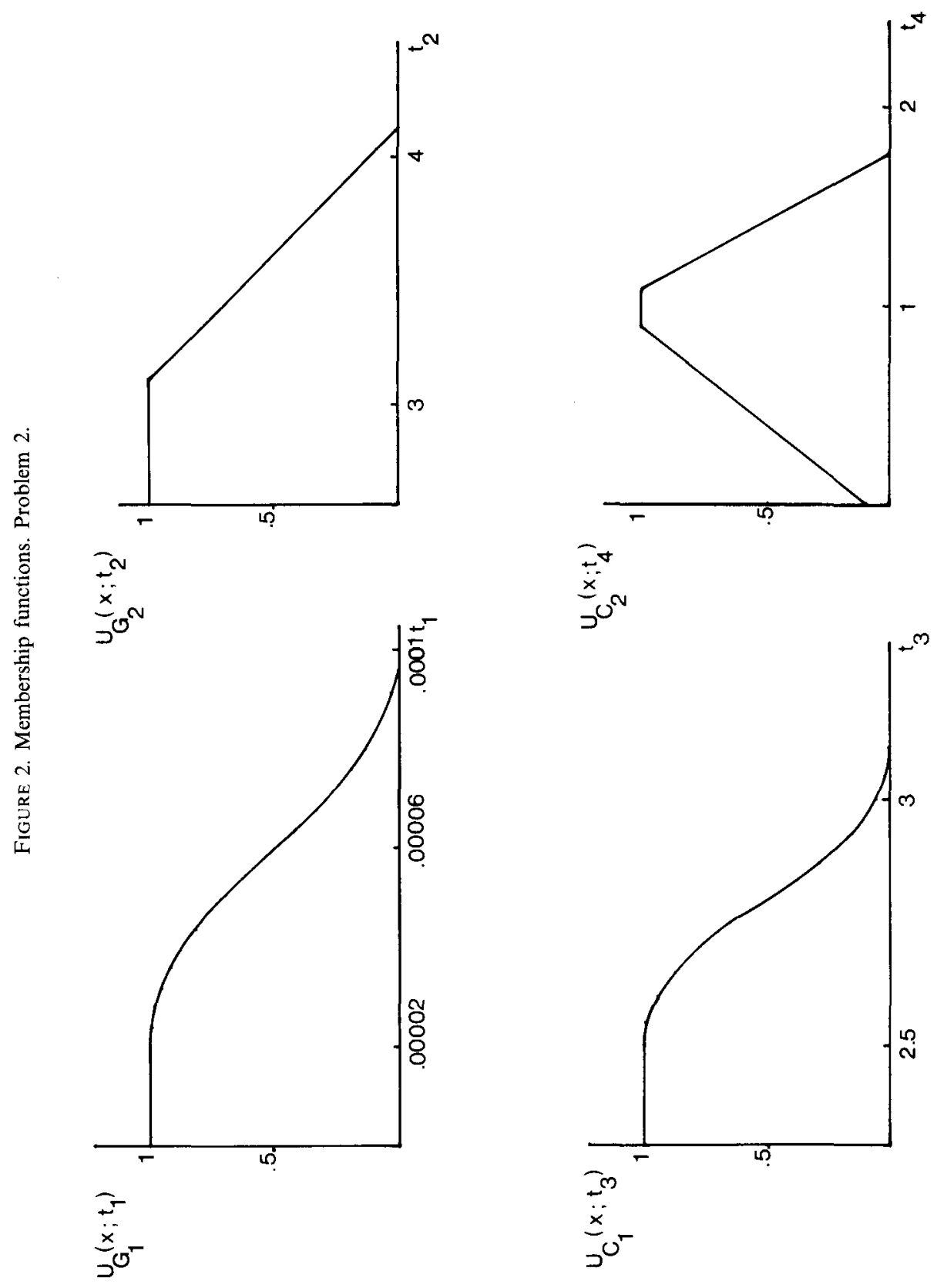
Constant 2 (deductible)

$$
U_{C_{2}}\left(x ; t_{4}\right)=\left\{\begin{array}{lc}
t_{4}+0.1 & 0<t_{4} \leqq 0.9 \\
1 & 0.9<t_{4} \leqq 1.1 \\
2.65-1.5 t_{4} & 1.1<t_{4} \leqq 1.7667 \\
0 & 1.7667<t_{4}
\end{array}\right.
$$

Given those membership functions, the grades of membership for all alternatives are easily computed. They are presented in Table 2.

TABLE 2

GRADES OF MEMBERSHIP OF THE 10 DIFFERENT PROGRAMS

\begin{tabular}{lcccccccccccc}
\hline \hline Program & 1 & 2 & 3 & 4 & 5 & 6 & 7 & 8 & 9 & 10 \\
\hline$G_{1}$ & .94 & .98 & 1 & 1 & .96 & .94 & .92 & .89 & .85 & .78 \\
$G_{2}$ & 1 & 1 & 1 & 1 & 1 & .91 & .82 & .58 & .30 & 0 \\
$C_{1}$ & 0 & 0 & .06 & .35 & .71 & .89 & .97 & .998 & 1 & 1 \\
$C_{2}$ & .5 & .7 & .9 & 1 & 1 & 1 & .85 & .55 & .25 & 0 \\
\hline
\end{tabular}

The membership function $U_{D}(x)$ of the decision $D$ is obtained by simply taking the minimum of the $U$ 's, for each alternative, as shown in Table 3.

TABLE 3

MEMBERSHIP FUNCTION OF $D$

\begin{tabular}{lllllllllll}
\hline \hline Program & 1 & 2 & 3 & 4 & 5 & 6 & 7 & 8 & 9 & 10 \\
\hline$U_{D}(x)$ & 0 & 0 & .06 & .35 & .71 & .89 & .82 & .55 & .25 & 0 \\
\hline
\end{tabular}

Note that no alternative has full membership in $D$ : fuzzy set $D$ is subnormal. This of course reflects the fact that the specified goals and constraints conflict with one another, ruling out the existence of an alternative which fully satisfies all of them.

In our case, when all goals and constraints are considered to be of equal importance, the ruin probability criterion is inoperative; it does not influence the decision. The membership function of $D$ is based on the first constraint for alternatives 1 to 6 , on the second goal for alternative 7 , and on the second constraint for alternatives 8 to 10 .

The optimal decision is program 6 , corresponding to a retention of $1.1 \%$ of the cedent's premium income. This alternative fully satisfies the second constraint, given our selection of membership functions. The other constraint and the two goals are conflicting and cannot be fully satisfied. The worst infringement is the reinsurance premium, considered to be too high.

Assume now that, after reviewing the preceding analysis, the manager of the reinsurance department decides that the first constraint $C_{1}$ is of paramount 
importance, and accordingly assigns it a higher weight. A concentration of the fuzzy set $C_{1}$, with $a=2$, is then performed : the values of $U_{C_{1}}\left(x ; t_{3}\right)$ are simply squared. This has the effect of decreasing the membership function of that important constraint and making it more influential in the determination of $D$. It is easily seen that the optimal decision becomes program 7 . This illustrates an inherent weakness of fuzzy decision-making: the sensitivity of the optimal solution to the particular selection of membership functions. And it is difficult to avoid an important element of subjectivity in the determination of those functions (see, however, Civanlar and Trussel (1986) and Dishkant (1981) for attempts to construct membership functions using statistical data).

The preceding analysis used the "hard" definition of the connective "and", since the minimum operator was used as intersection. As illustrated in Example 1, this excludes all forms of compensations and interactions between the goals and constraints. In some managerial problemes the decision maker might wish to be less restrictive. For instance, he might not really want to actually maximize the objective function, but rather reach some aspiration level, which might not even be definable crisply (his objective might be to "improve the present cost situation considerably", for instance). Or the " $\leqq$ " sign in a constraint might not be meant in the strict mathematical sense, but small violations might be acceptable, especially if an important improvement in the objective function results (effective expenditures might slightly exceed a budget constraint, for instance). Hence in many cases it is more appropriate to use a "softer" aggregation operator than the minimum, like the bounded difference or the Yager operator. A decision is then defined as the confluence of goals and constraints.

$$
U_{D}(x)=U_{G_{1}}(x) * \ldots * U_{G_{p}}(x) * U_{C_{1}}(x) \ldots * U_{C_{q}}(x),
$$

where $*$ is the selected operator.

It is easily checked, for instance, that if the algebraic product is used instead of the minimum operator, program 6 is the optimal solution of problem 2, with program 5 a close second.

\section{FUZZY ARITHMETICS AND FUZZY INSURANCE PREMIUMS}

Definitions. A fuzzy number is a fuzzy subset of the real line whose highest membership values are clustered around a given real number. The membership function is monotonic on both sides of this real number. More precisely, a fuzzy number $A$ is a fuzzy subset of the real line $R$ whose membership function $U_{A}(x)=U_{A}\left(x ; a_{1}, a_{2}, a_{3}, a_{4}\right)$ is:

(i) a continuous mapping from $R$ to the closed interval $[0,1]$

(ii) zero on the interval $\left(-\infty, a_{1}\right]$

(iii) strictly increasing on the interval $\left[a_{1}, a_{2}\right]$

(iv) one on the interval $\left[a_{2}, a_{3}\right]$

(v) strictly decreasing on the interval $\left[a_{3}, a_{4}\right]$

(vi) zero on the interval $\left[a_{4}, \infty\right)$, 
where $a_{1}<a_{2}<a_{3}<a_{4}$. (Examples of membership functions of fuzzy numbers are presented in Figure 3). The increasing part of $U_{A}(x)$, on interval $\left[a_{1}, a_{2}\right]$, is denoted $U_{A 1}(x)$; the decreasing part of $U_{A}(x)$, on interval $\left[a_{3}, a_{4}\right]$, is denoted $U_{A 2}(x)$. Alternatively, the inverse functions of $U_{A 1}(x)$ and $U_{A 2}(x)$, $U_{A 1}^{-1}(y)$ and $U_{A 2}^{-1}(y)$ can be used; they are denoted $V_{A 1}(y)$ and $V_{A 2}(y)$.

If $a_{1}=a_{2}=a_{3}=a_{4}, A$ is an ordinary real number.

A fuzzy number $A$ is said to be positive if $a_{1}>0$. It is negative if $a_{4}<0$.

Let $A$ and $B$ be two fuzzy numbers with membership functions $U_{A}(x)=U_{A}\left(x ; a_{1}, a_{2}, a_{3}, a_{4}\right)$ and $U_{B}(x)=U_{B}\left(x ; b_{1}, b_{2}, b_{3}, b_{4}\right)$. The membership function of the sum $C$ of $A$ and $B$, denoted $A \oplus B$, is defined as

$$
\begin{aligned}
U_{C}(z) & =\max _{x+y=z} \min \left[U_{A}(x), U_{B}(y)\right] \quad(x, y, z) \in R^{3} \\
& =\max \min \left[U_{A}(x), U_{B}(z-x)\right] .
\end{aligned}
$$

It can be shown (see for instance DUBoIS and PRADE (1978) and (1980)) that the sum of fuzzy numbers is associative and commutative, and that

(i) $U_{C}(z)=0$

$$
z \in\left(-\infty, a_{1}+b_{1}\right] U\left[a_{4}+b_{4}, \infty\right]
$$

(ii) $U_{C}(z)$ is strictly increasing in $\left[a_{1}+b_{1}, a_{2}+b_{2}\right]$, and strictly decreasing in $\left[a_{3}+b_{3}, a_{4}+b_{4}\right]$

(iii) $U_{C}(z)=1$

$$
z \in\left[a_{2}+b_{2}, a_{3}+b_{3}\right]
$$

(iv) $U_{C 1}(z)=\left[U_{A 1}^{-1}(z)+U_{B 1}^{-1}(z)\right]^{-1}$ or $V_{C 1}(z)=V_{A 1}(z)+V_{B 1}(z)$

$$
U_{C 2}(z)=\left[U_{A 2}^{-1}(z)+U_{B 2}^{-1}(z)\right]^{-1} \text { or } V_{C 2}(z)=V_{A 2}(z)+V_{B 2}(z) \text {. }
$$

The product $D$ of $A$ and $B$, denoted $A \odot B$, is defined by

$$
U_{D}(z)=\max _{x y=z} \min \left[U_{A}(x), U_{B}(y)\right] \quad \text { (assuming } a_{1}, b_{1}>0 \text { ) }
$$

It can be shown that $D$ is a fuzzy number, with $d_{1}=a_{1} b_{1}, d_{2}=a_{2} b_{2}$, $d_{3}=a_{3} b_{3}, d_{4}=a_{4} b_{4}$,

$$
\begin{array}{llll}
U_{D 1}(z)=\left[U_{A 1}^{-1}(z) \cdot U_{B 1}^{-1}(z)\right]^{-1} & \text { or } & V_{D 1}(z)=V_{A 1}(z) \cdot V_{B 1}(z) \\
U_{D 2}(z)=\left[U_{A 2}^{-1}(z) \cdot U_{B 2}^{-1}(z)\right]^{-1} & \text { or } & V_{D 2}(z)=V_{A 2}(z) \cdot V_{B 2}(z) .
\end{array}
$$

The product is associative and commutative, and distributive on $\oplus$. The $n^{\text {th }}$ power of $A$ is naturally recursively defined as

$$
A^{n}=A \odot A^{n-1}
$$

The only reference dealing with finance applications of fuzzy arithmetics seems to be BUCKLEY (1987), who defined the fuzzy extensions of the notions of present and accumulated value, and annuities, and showed how to compare fuzzy cash flows by means of extended net present value and internal rate of return methods. Problem 3 is a straightforward generalization of that paper to an insurance problem. 


\section{Problem 3}

Let us compute the net single premium of a $\$ 1000,10$-year pure endowment policy, on a life aged (55), where $p={ }_{10} p_{55}$ is 0.87 . The interest rate $i$ is fuzzy and assumed to be approximately equal to $6 \%$, as modelized by

$$
U_{i}(x)=\left\{\begin{array}{lr}
0 & x \leqq 1.03 \\
U_{i 1}(x)=50 x-51.5 & 1.03<x \leqq 1.05 \\
1 & 1.05<x \leqq 1.07 \\
U_{i 2}(x)=54.5-50 x & 1.07<x \leqq 1.09 \\
0 & 1.09<x
\end{array}\right.
$$

(see Figure 3, upper left). As shown by the definitions of $\oplus$ and $\odot$, it is easier to use the inverse functions

$$
V_{i 1}(y)=1.03+0.02 y \text { and } V_{i 2}(y)=1.09-0.02 y .
$$

The present value $P V(S, n)$ of a positive fuzzy amount $S, n$ periods in the future, if the fuzzy interest rate is $i$ per period, can be defined as

$$
P V(S, n)=S \odot(1 \oplus i)^{-n}
$$

This definition makes sense given the associativity and the distributivity properties of $\odot$. Note however that, generally, $P V(S, n) \odot(1 \oplus i)^{n}$ will not be equal to $S$. Since the face value and the survival probability are nonfuzzy, the single fuzzy premium $A$ of the policy,

$$
A=1000 \cdot 0.87 \cdot(1 \oplus i)^{-10},
$$

is defined by the membership function

$$
U_{A}(x)=\left\{\begin{array}{lr}
0 & x \leqq 367.50 \\
U_{A 1}(x) \text { or } V_{A 1}(y) & 367.50<x \leqq 442.26 \\
1 & 442.26<x \leqq 534.10 \\
U_{A 2}(x) \text { or } V_{A 2}(y) & 534.10<x \leqq 647.36 \\
0 & 647.36<x
\end{array}\right.
$$

where

$$
V_{A 1}(y)=870(1.09-0.02 y)^{-10}
$$

and

$$
V_{A 2}(y)=870(1.03+0.02 y)^{-10} \quad(0 \leqq y \leqq 1)
$$

This function is represented in Figure 3, upper right. 

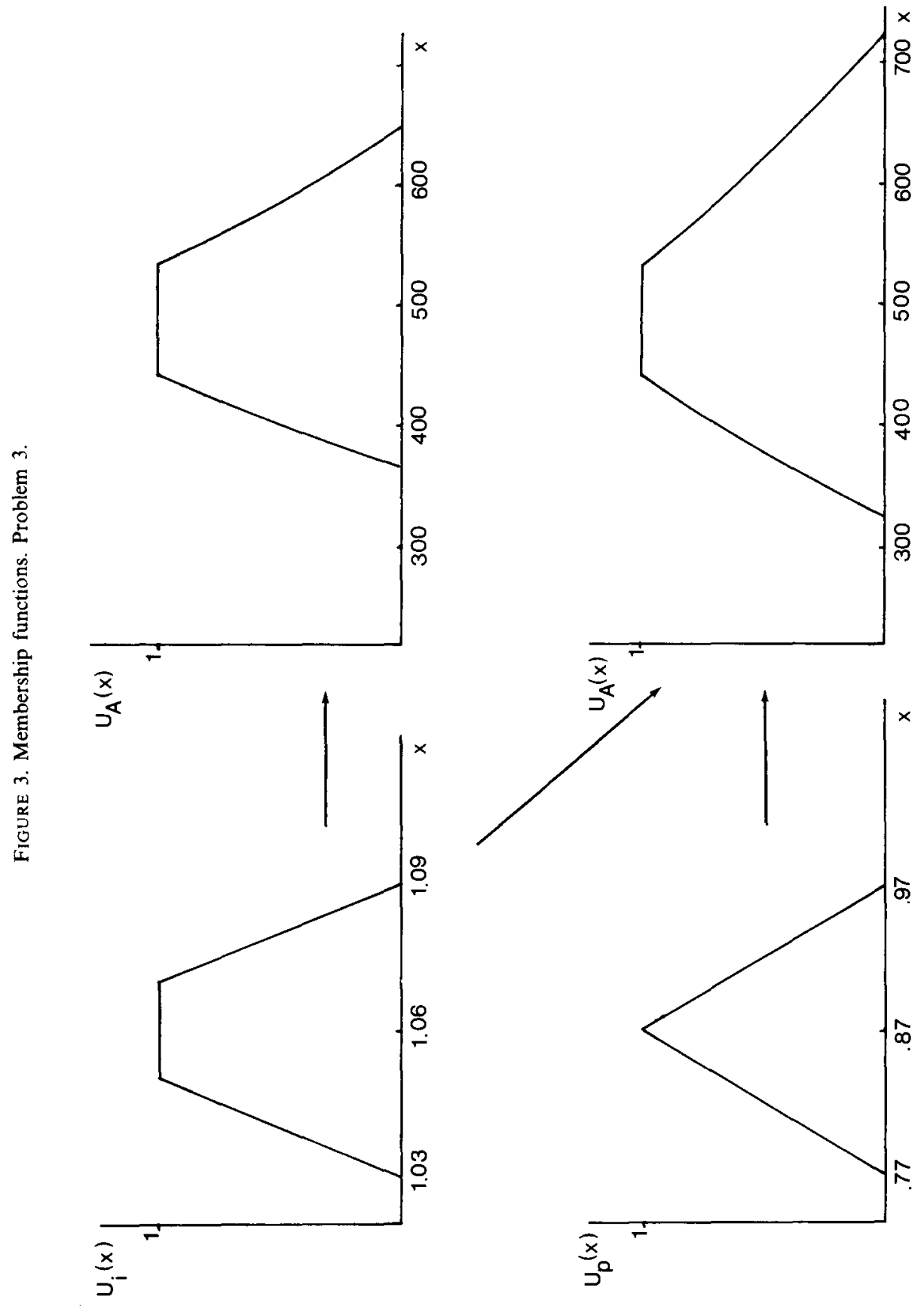
Next assume that $p={ }_{10} p_{55}$ is also fuzzy, with membership function

$$
U_{p}(x)=\left\{\begin{array}{lc}
0 & (x \leqq 0.77) U(x>0.97) \\
10 x-7.7 & 0.77<x \leqq 0.87 \\
9.7-10 x & 0.87<x \leqq 0.97
\end{array}\right.
$$

and inverse functions $V_{p 1}(y)=0.77+0.01 y$ and $V_{p 2}(y)=0.97-0.01 y$ (see Figure 3, lower left).

The membership function of the premium $A$ now becomes

$$
U_{A}(x)=\left\{\begin{array}{lr}
0 & x \leqq 325.26 \\
U_{A 1}(x) \text { or } V_{A 1}(y) & 325.26<x \leqq 442.26 \\
1 & 442.26<x \leqq 534.10 \\
U_{A 2}(x) \text { or } V_{A 2}(y) & 534.10<x \leqq 721.77 \\
0 & 721.77<x
\end{array}\right.
$$

where

$$
\begin{aligned}
& V_{A j}(y)=1000 \cdot V_{p j}(y) \cdot\left[1+V_{i, 3-j}(y)\right]^{-10} \quad j=1,2 \\
& V_{A 1}(y)=1000(0.77+0.1 y)(1.09-0.02 y)^{-10} \\
& V_{A 2}(y)=1000(0.97-0.1 y)(1.03+0.02 y)^{-10}
\end{aligned}
$$

This membership function, represented in the lower right part of Figure 3, reflects the increased fuzziness.

It is also possible (see BUCKLEY (1987)) to fuzzify the number of periods $n$.

\section{FUZZY SETS LITERATURE}

The literature about fuzzy sets is abundant and highly specialized. A good introductory textbook is ZIMMERMANN (1987), despite the important number of misprints. More specialized textbooks are KAUFMANN (1975) and DUBoIS and PRADE (1980). The seminal papers about fuzzy decision-making are Bellman and Zadeh (1970) and YAGer and Besson (1976). Fuzzy graph theory, fuzzy linear and dynamic programming and extensions of other operations research methods are surveyed in ZIMMERMANN (1985) and (1987). Reference papers for applications of fuzzy set theory to statistics are HESHMATY and KANDER (1985), BUCKLEY (1985) and JAJUGA (1986). Topics of interest for actuaries where fuzzy applications have been developed include game theory (AUBIN (1981), BUTNARIU (1978, 1980)), economics (Chang (1977), Chen et al. (1980)), and utility theory (MAthieuNiCOT (1986)). 


\section{REFERENCES}

Aubin, J.P. (1981) Cooperative Fuzzy Games. Math. Oper. Res. 6, 1-13.

Bellman, R. and ZaDeH, L. (1970) Decision-making in a Fuzzy Environment. Management Science 17, B141-B164.

Buckley, J. (1985) Fuzzy Decision Making with Data : Applications to Statistics. Fuzzy Sets and Systems 16, $139-147$.

BuCKLey, J. (1987) The Fuzzy Mathematics of Finance. Fuzzy Sets and Systems 21, 257-273.

Butnariu, D. (1978) Fuzzy Games: A Description of the Concepts. Fuzzy Sets and Systems 1, 181-192.

ButNariu, D. (1980) Stability and Shapley Value for an $n$-persons Fuzzy Game. Fuzzy Sets and Systems 4, 63-72.

Chang, S. (1977) Application of Fuzzy Set Theory to Economics. Kybernetics 6, $203-207$.

Chen, G., LeE, S. and YU, E. (1980) Application of Fuzzy Set Theory to Economics. In Advances in Fuzzy Sets, Possibility Theory, and Applications, (ed. P. Wang) pp. 227-305, Plenum Press, New York.

Civanlar, R. and Trussell, J. (1986) Constructing Membership Functions Using Statistical Data. Fuzzy Sets and Systems 18, 1-13.

Dishkant, H. (1981) About Membership Functions Estimations. Fuzzy Sets and Systems 5, 141-147.

Dubors, D. and Prade, H. (1978) Operations on Fuzzy Numbers. Int. J. Systems Sci. 9, 613-626.

Dubors, D. and Prade, H. (1980) Fuzzy Sets and Systems. Academic Press. New York.

Gupta, M., Saridis, G. and Gaines, B., Editors. (1977) Fuzzy Automata and Decision Processes. North Holland, Amsterdam.

Heshmaty, B. and Kander, A. (1985) Fuzzy Linear Regression and its Applications to Forecasting in Uncertain Environment. Fuzzy Sets and Systems 15, 159-191.

Jajuga, K. (1986) Linear Fuzzy Regression. Fuzzy Sets and Systems, 20, 1986, 343-353.

Kaufmann, A. (1975) Introduction to the Theory of Fuzzy Subsets. Vol I. Academic Press, New York.

Mathieu-Nicot, B. (1986) Fuzzy Expected Utility. Fuzzy Sets and Systems 19, 163-173.

YAGER, R. and Basson, D. (1976) Decision-making with Fuzzy Sets. Decision Sciences 6, $590-600$.

ZaDeH, L. (1965) Fuzzy Sets. Inf. and Control 8, 338-353.

Zadeh, L., Fu, K., Tanaka, K. and Shimura, M., Editors. (1975) Fuzzy Sets and Their Applications to Cognitive and Decision Processes. Academic Press, New York.

Zimmermann, H-J. (1985) Fuzzy Set Theory and its Applications. Kluwer, Boston.

ZimmermanN, H-J. (1987) Fuzzy Sets, Decision Making and Expert Systems. Kluwer, Boston.

JEAN LEMAIRE

Insurance Department, Wharton School, 3641 Locust Walk, University of Pennsylvania, Philadelphia, PA 19104, U.S.A. 